\title{
A Laser-Diode-Pumped Nd: Glass Laser: Mode-Locked, High Power, and Single Frequency Performance
}

\author{
David W. Hughes, Mark W. Phillips, John R. M. Barr, and David C. Hanna
}

\begin{abstract}
We report the performance of a laser-diodepumped Nd:glass laser. Using the $A M$ active mode-locking technique, pulses as short as 9 ps were obtained. The development of a high power $(415 \mathrm{~mW})$, high optical slope efficiency $(32.4 \%)$ laser-diode-pumped Nd : glass laser using high power, broad stripe laser diodes as the pump source is described. Single frequency operation of the Nd: glass laser, using an acoustooptic modulator to ensure unidirectional operation in a ring laser configuration was investigated. An instantaneous linewidth of less than $300 \mathrm{~Hz}$ was observed.
\end{abstract}

\section{INTRODUCTION}

$\mathrm{N}$ EODYMIUM-DOPED glass is of great interest as a laser-diode-pumped laser medium. $\mathrm{Nd}$ : glass has a strong absorption in the region of $800 \mathrm{~nm}$, and so can be readily pumped by commercially available GaAlAs laser diodes. The pump absorption bands of $\mathrm{Nd}$ : glass are much broader than those of Nd:YAG or Nd:YLF, so that stringent wavelength control of the pump source via temperature tuning is unnecessary. Traditionally, the $\mathrm{Nd}$ : glass laser has been pumped using flashlamp pumping or end pumping using an argon laser operating at $514 \mathrm{~nm} \mathrm{[1].}$ The greater compactness and higher efficiency of the laser diode over these other pump sources has resulted in a number of recent publications concerning the laser-diodepumped Nd: glass laser [2]-[7]. Unlike many other laser hosts, the concentration of neodymium ions can be very high in glass before the onset of severe fluorescence quenching (typically $8 \mathrm{wt} \%$ in glass as opposed to 1.3 wt $\%$ in YAG). This means that more compact laser resonators can be designed.

The broad fluorescence linewidth of $\mathrm{Nd}$ :glass $(5.3$ THz, $19.6 \mathrm{~nm}$ for Schott LG760 Nd-doped phosphate glass) means that the $\mathrm{Nd}$ : glass laser is capable of supporting subpicosecond mode-locked pulses. Several authors [3]-[6] have reported active mode locking of the laser-diode-pumped Nd: glass laser using both amplitude modulation (AM) [3]-[5] and frequency modulation (FM) [6] techniques. The Nd: glass laser has also been mode

\footnotetext{
Manuscript received July 17, 1991; revised September 23, 1991. This work was supported in part by the Science and Engineering Research Council (SERC) by Grant GR/F 40658, the Opto-Electronic Research Centre, and the Nuffield Foundation. The work of D. W. Hughes was supported by the SERC.

The authors are with the Opto-Electronic Research Centre, Southampton University, Southampton, SO9 5NH U.K.

IEEE Log Number 9106682
}

locked using the technique of additive-pulse mode locking (APM) [8]. In the latter case, a krypton ion laser was used as the pump source in order to achieve the necessary output power from the $\mathrm{Nd}$ : glass laser for this technique to be self starting. In Section II we present the performance of a laser-diode-pumped mode-locked Nd : glass laser. Acoustooptic amplitude modulation (AM) mode locking was used, yielding a pulse duration of $9 \mathrm{ps}$.

The main disadvantage of Nd: glass as compared with $\mathrm{Nd}$ : YAG is its low thermal conductivity $(0.6 \mathrm{~W} / \mathrm{m} / \mathrm{K}$ as opposed to $11 \mathrm{~W} / \mathrm{m} / \mathrm{K}$ ). This leads to a number of deleterious effects, including thermally induced birefringence, thermal lensing and physical thermal damage. These problems have, in the past, largely restricted the performance of the laser-diode-pumped $\mathrm{Nd}$ : glass laser to relatively low output powers [4]-[6]. Korn et al. [7] have surmounted this problem to some extent by using a rotating $\mathrm{Nd}$ : glass disk as the gain medium, obtaining output powers as high as $550 \mathrm{~mW}$ with an optical slope efficiency of $37 \%$. In Section III we will discuss the use of high power broad stripe laser diodes as an alternative means of obtaining high efficiency, high power operation of the $\mathrm{Nd}$ : glass laser with a stationary gain medium. This technique was found to produce a similar output to that obtained using a $\mathrm{Ti}$ : sapphire laser as the pump source.

We have also obtained interesting results from a single frequency $\mathrm{Nd}$ : glass laser, pumped by a $\mathrm{Ti}$ : sapphire laser at $805 \mathrm{~nm}$. Using an acoustooptic modulator as the means of obtaining unidirectional operation [9] from a $\mathrm{Nd}$ : glass ring laser, a linewidth of less than $300 \mathrm{~Hz}$ has been obtained. To our knowledge this is the narrowest linewidth reported using this technique. The results from this experiment will be briefly reported in Section IV, and it is emphasized that this system is expected to work equally well when pumped with laser diodes.

\section{The Mode-Locked Laser-Diode-Pumped Nd: Glass Laser}

The mode-locked Nd: glass laser has, as stated previously, been pumped using both flashlamp pumping and end pumping with an argon ion laser. Using the latter approach, Yan et al. [1] obtained pulses as short as 10 ps from an AM mode-locked Nd: glass laser. Several authors [3]-[6] have more recently reported the performance of actively mode-locked laser-diode-pumped $\mathrm{Nd}$ :glass 


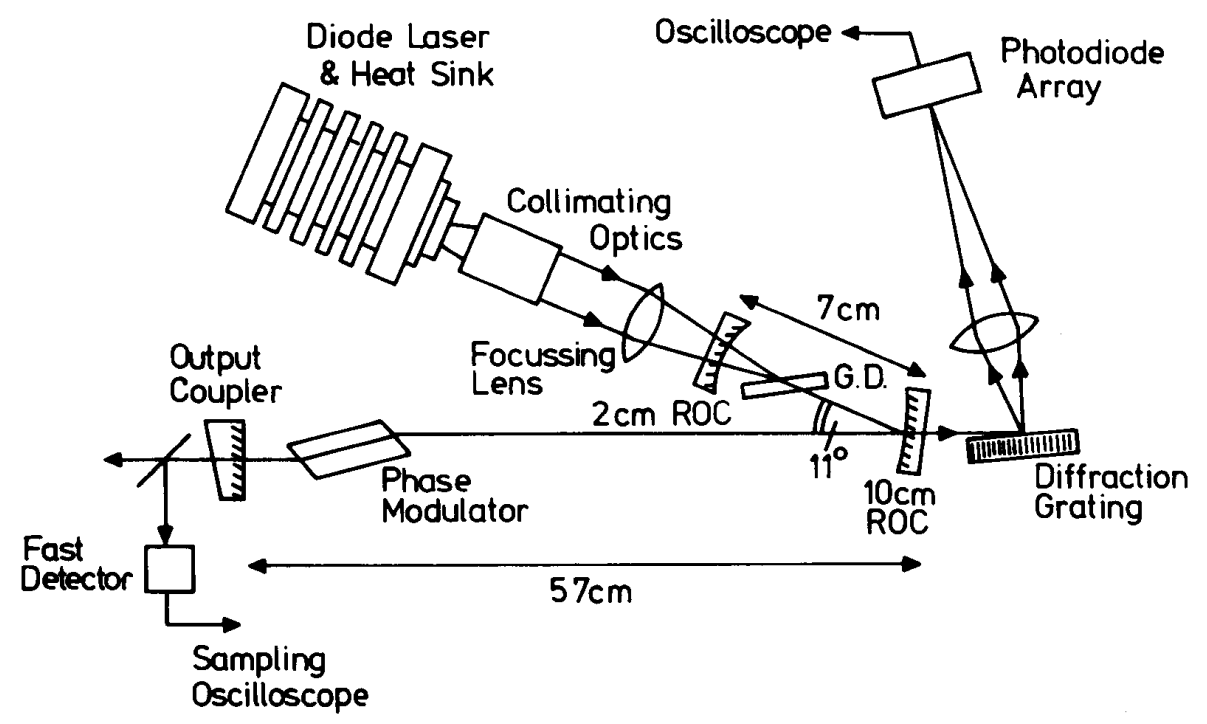

Fig. 1. The mode-locked laser-diode-pumped Nd: glass laser. The laser is shown configured for FM mode locking. GD: Nd: glass disk.

lasers, obtaining pulse durations ranging from $7 \mathrm{ps}[4]$ to $58 \mathrm{ps}$ [5]. In this section, we present results obtained from an actively mode-locked laser-diode-pumped $\mathrm{Nd}$ : glass laser, where the AM mode-locking technique has been used.

Fig. 1 shows a schematic of the laser used in these experiments. The pump source was a $500 \mathrm{~mW}$, ten stripe laser diode array (SDL 2432). This was mounted on a heatsink, and temperature tuned using a Peltier cooler in order to achieve maximum absorption of the pump radiation in the active medium. The laser diode output was collimated using a compound lens of focal length $6.5 \mathrm{~mm}$ and numerical aperture 0.6 and passed through an anamorphic prism beam expander (magnification $\times 5$ ). The beam was then expanded using a $2 \times$ telescope in order to exclusively pump the $\mathrm{TEM}_{00}$ mode of the $\mathrm{Nd}$ : glass laser. The pump beam was then focused through the laser cavity rear mirror using a $3.2 \mathrm{~cm}$ focal length lens. The collimating and focusing optics were all antireflection (AR) coated at the pump wavelength. The active medium was a $1.2 \mathrm{~mm}$ thick, $10 \mathrm{~mm}$ diameter disk of highly doped (8 wt\%) LG760 Nd : phosphate glass. This thickness of glass was sufficient to absorb more than $90 \%$ of the pump radiation. A low insertion loss was achieved by placing the disk at Brewster's angle at the focus of a three mirror astigmatically compensated cavity [10], and was held between two copper plates to aid heat removal. The copper plates were $30 \mathrm{~mm} \times 20 \mathrm{~mm}$ in dimension, with a clear aperture of $8 \mathrm{~mm}$. The $\mathrm{Nd}$ : glass disk was pumped at its center. The required angle of incidence on the $10 \mathrm{~cm}$ radius of curvature (ROC) turning mirror for compensation of the astigmatism produced by the Brewster angled disk was calculated to be $5.5^{\circ}$. The average $\mathrm{TEM}_{00}$ mode size (FWHM) in the active medium was calculated to be 21 $\mu \mathrm{m}$, taking into account the refractive index of the active medium. The focused pump spot size (FWHM) was 24 $\mu \mathrm{m}$ in the plane of the array and $<24 \mu \mathrm{m}$ in the plane perpendicular to the array (the resolution limit of our measurement was $24 \mu \mathrm{m}$ ). The cavity rear mirror (ROC $2 \mathrm{~cm}$ ) and the turning mirror were highly reflective (HR) at 1.05 $\mu \mathrm{m}$ ( $>99.9 \%$ reflectivity) and highly transmissive at 800 nm ( $>90 \%$ transmission). The output coupler had a re flectivity of $98.5 \%$, and a wedge angle of $10^{\circ}$. The low output coupling was chosen due to the low gain of the $\mathrm{Nd}$ : glass laser system, and to allow the insertion of lossy elements (phase and amplitude modulators) into the cavity. The rear surface of each of the cavity mirrors was AR coated at $1.05 \mu \mathrm{m}$ to suppress unwanted etalon effects.

With no modulator in the cavity, the laser exhibited a threshold of $60 \mathrm{~mW}$ absorbed pump power, and an optical slope efficiency of $11 \%$. This slope efficiency is quoted for pump powers below $200 \mathrm{~mW}$. Above $200 \mathrm{~mW}$, the slope efficiency decreased with pump power. We have attributed this decrease to thermally induced birefringence [11].

Amplitude modulation mode locking of the Nd:glass laser was accomplished using an acoustooptic standingwave modulator (Isle Optics ML120B). This was a Brewster angled fused quartz device placed as close as possible to the laser output coupler. The modulator was driven at a radio frequency (RF) of $120 \mathrm{MHz}$ using an RF synthesizer and broad-band RF amplifier. This corresponded to a laser cavity length of $62.5 \mathrm{~cm}$. With an RF power of $1 \mathrm{~W}$, the diffraction efficiency of the device was measured to be $18.2 \%$. The diffraction efficiency $\epsilon$ is related to the modulation depth $\theta$ by the expression

$$
\epsilon=0.5\left[1-J_{0}(2 \theta)\right]
$$

where $J_{0}(x)$ is the zero-order Bessel function of $x$. The modulation depth was thus calculated to be 0.63 at a drive 
power of $1 \mathrm{~W}$. The passive insertion loss of the device in the laser cavity was negligible. However, when the laser was mode locked, the output power was found to drop to about $30 \mathrm{~mW}$ (for maximum pump power), which was a decrease of about $15 \%$ from the output when the laser was not mode locked.

The mode-locked laser output was observed using a fast photodetector (GE Y-35-5252 $25 \mathrm{GHz}$ photodiode) and a Tektronix sampling scope. The observed pulse duration (50 ps) was seen to be limited by the response of this combination. For more accurate measurements, a standard nonbackground free-autocorrelation technique was used. The moving prism in the autocorrelator was scanned at a rate of up to several Hertz. A KTP crystal was used for second harmonic generation. A typical autocorrelation trace is shown in Fig. 2. If we assume a Gaussian temporal profile, the width of 13 ps (FWHM) of this autocorrelation trace corresponds to an optical pulse duration of 9 ps. This pulse duration was observed for an RF drive power of $0.8 \mathrm{~W}$. The diffraction efficiency of the modulator at this power was $13.8 \%$. It should be noted that for 9 ps pulses to be obtained, extremely careful adjustment of the orientation of the modulator had to be carried out in order to optimize the Bragg angle.

In a previous paper, Krausz et al. [4] have reported slighter shorter pulses ( $7 \mathrm{ps}$ ) than those reported here from a similar laser-diode-pumped AM mode-locked Nd : glass laser. However, in that paper, the shortest (7 ps) pulses were only observed at pump powers slightly above threshold. At higher pump powers (corresponding to an average output power of $40 \mathrm{~mW}$ ) the pulse duration increased to 20 ps. The authors suggested that this may be due to selfphase modulation (SPM) in the active medium. In our experiments, we have observed no such change in the pulse duration with pump power.

The optical spectrum of the laser was monitored using a 1800 lines $/ \mathrm{mm}$ diffraction grating spectrometer. The free-running spectrum had a FWHM of $7.5 \mathrm{GHz}$ as shown in Fig. 3(a). When the laser was mode locked such that the 9 ps pulses were obtained, the spectrum FWHM increased to $80 \mathrm{GHz}$ as shown in Fig. 3(b). This yields a time-bandwidth product of 0.72 which is 1.6 times transform limited. Unlike in the work of Krausz et al. [4], we observed no modulated structure on the mode-locked optical spectrum. We did, however, observe heavily modulated and shifted optical spectra as the modulator frequency was detuned from its optimum mode-locking value (i.e., the value which yielded the shortest pulse).

We have previously reported FM mode locking of this laser [6], where we also obtained 9 ps pulses. We have thus observed no significant difference between the pulse durations obtained using AM and FM mode locking. This is in contrast to the results of Maker et al. [12], [13] who carried out experiments on the active mode locking of a laser-diode-pumped Nd:YAG laser. They obtained significantly shorter pulses for FM mode locking than for AM mode locking (12 ps as opposed to $55 \mathrm{ps).} \mathrm{The} \mathrm{reason}$ for this was not fully understood. One point which should

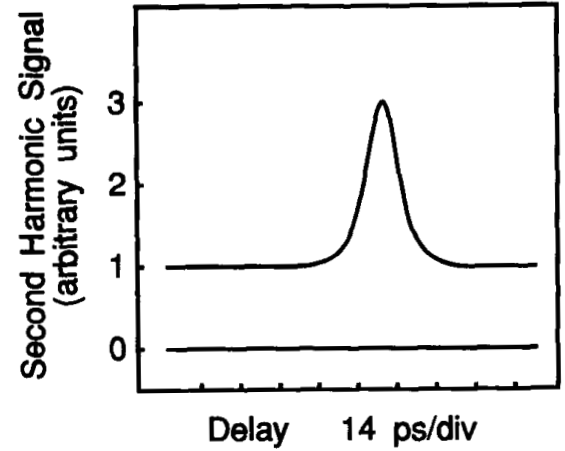

Fig. 2. A typical AM mode-locked autocorrelation trace of duration 13 ps.

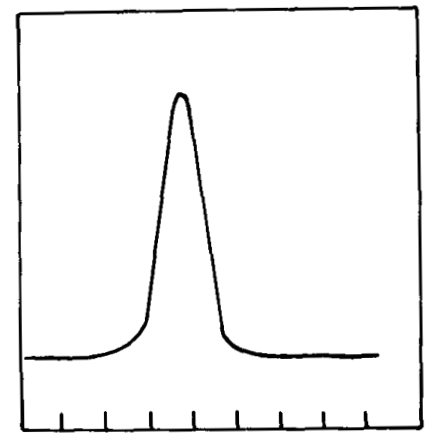

(a)

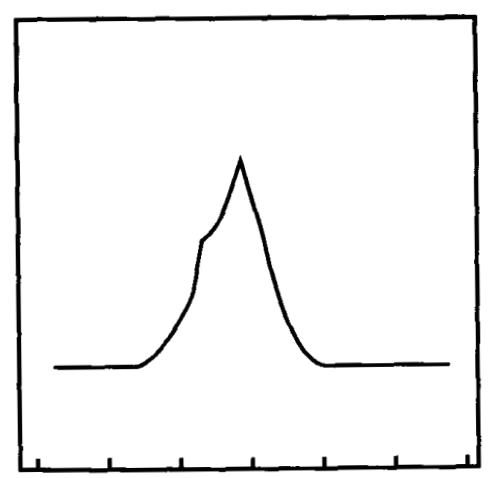

(b)

Fig. 3. (a) Free running laser spectrum. Scale $7.5 \mathrm{GHz} / \mathrm{div}$. (b) $\mathrm{AM}$ modelocked laser spectrum. Scale $75 \mathrm{GHz} /$ div.

be noted, however, is that the laser cavity used for the Nd: YAG laser was markedly different to ours, with the gain medium adjacent to the cavity rear mirror. This leads to a much broader free-running linewidth than we have seen in our experiments. This broadening has been attributed to spatial hole burning [14].

\section{High Power Operation of the Laser-Diode- Pumped Nd: Glass Laser}

As was mentioned in the introduction, one of the main disadvantages of the $\mathrm{Nd}$ : glass laser is its low thermal 


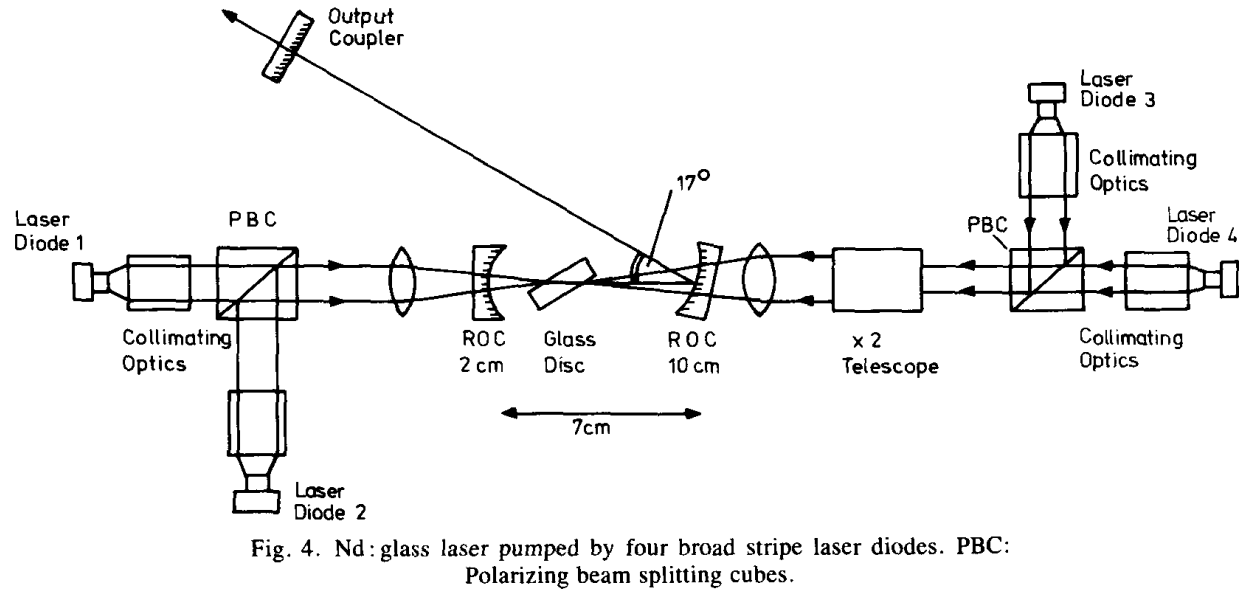

conductivity, which restricts high power operation. High efficiency operation ( $42 \%$ optical slope efficiency) has been reported using a single stripe laser diode as the pump source [2]. Due to the low power of the pump source, however, the laser output did not exceed a few milliwatts. Higher output powers (tens of milliwatts) have been reported using laser diode arrays as pump sources [4]-[6], but this in turn leads to lower slope efficiencies due to the poorer match between the focused pump beam and the $\mathrm{Nd}$ : glass laser cavity mode. The problem is compounded by thermal considerations, especially thermally induced birefringence and thermal physical damage [11]. Thermally induced birefringence causes a decrease in the laser efficiency as the pump power is increased, for a laser with a polarization selective element. We have also found that the highly doped glass used in our initial experiments melted at the pump input face at a pump power of about $600 \mathrm{~mW}$ (obtained by polarization coupling two $500 \mathrm{~mW}$ laser diodes) so that no significant increase in output power could be achieved simply by increasing the pump power. In this section we describe the performance and design of a laser-diode-pumped Nd : glass laser which has an output power of $415 \mathrm{~mW}$ and an optical slope efficiency of $32.4 \%$.

The incentive behind this work is again to obtain short mode-locked pulses. There is currently much interest in the technique of additive pulse mode locking (APM). This is a technique in which a portion of the laser output is directed into an external cavity containing some nonlinear element. This portion of the output suffers a nonlinear phase shift or a nonlinear amplitude change, and, provided the cavity lengths are properly matched, can interfere interferometrically with a pulse circulating in the main laser cavity. This can result in the pulse in the main laser cavity being shortened. This technique has been shown to be self-starting in several laser systems, e.g., Nd : YAG [15], [16], Nd : YLF [17], and Nd: glass [8]. In each case, an intensity dependent threshold has been observed for the onset of self-starting APM. Krausz et al. [8] reported that a laser output of about $250 \mathrm{~mW}$ was nec- essary for stable mode locking to be achieved in the $\mathrm{Nd}$ : glass laser (it is thus necessary to increase dramatically the powers obtained from the laser-diode-pumped $\mathrm{Nd}$ : glass laser if subpicosecond APM operation is to be achieved from this all solid-state system).

We have previously reported the use of high power broad stripe laser diodes to pump the Nd: glass laser described in Section II [11]. Two $500 \mathrm{~mW}$ broad stripe laser diodes (STC LQ-(P)-05) pumped the laser, one through the cavity rear mirror and the other through the turning mirror. This double end pumping allowed us to obtain a more uniform longitudinal heat distribution throughout the glass disk, and with more careful heatsinking to reduce the effects of thermally induced birefringence, we obtained an output power of $153 \mathrm{~mW}$ (with an output coupling of 5\%). However, no further significant increase in output power could be obtained due to the glass melting.

To combat this problem, and to enable us to pump the laser with four laser diodes, we have carried out experiments using a lower doped glass ( $4 \mathrm{wt} \%)$ which gives an increased volume in which the heat is dissipated. The experimental arrangement is shown in Fig. 4. The collimating optics for all four diodes were identical to that described in Section II, but a prism beam expansion of $\times 6$ was used. Laser diodes three and four were focused through the turning mirror using an AR coated lens of focal length $7.5 \mathrm{~cm}$. A $2 \times$ telescope was used to expand the beams from laser diodes three and four to ensure single transverse mode operation of the $\mathrm{Nd}$ : glass laser. The $2.4 \mathrm{~mm}$ thick glass disk (this thickness was sufficient to absorb $95 \%$ of the incident pump radiation) was held between two copper plates, and indium foil (75 $\mu \mathrm{m}$ thick) was placed between the glass and the copper to ensure good thermal contact. The copper plates were as described previously, but the clear aperture was now reduced to less than $3 \mathrm{~mm}$. The angle of incidence of the turning mirror was now increased to $8.4^{\circ}$ to take into account the increased thickness of the glass disk. The average Nd: glass laser mode size (FWHM) in the active medium was calculated to be $22 \mu \mathrm{m}$. The focused pump spot 


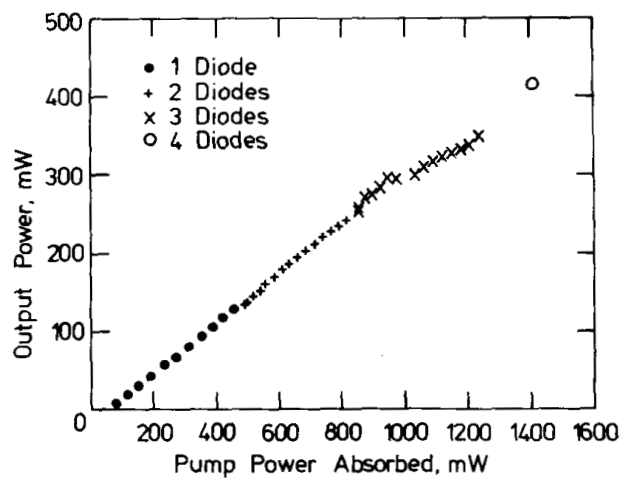

Fig. 5. Laser-diode-pumped $\mathrm{Nd}$ : glass laser performance with $5 \%$ output coupling.

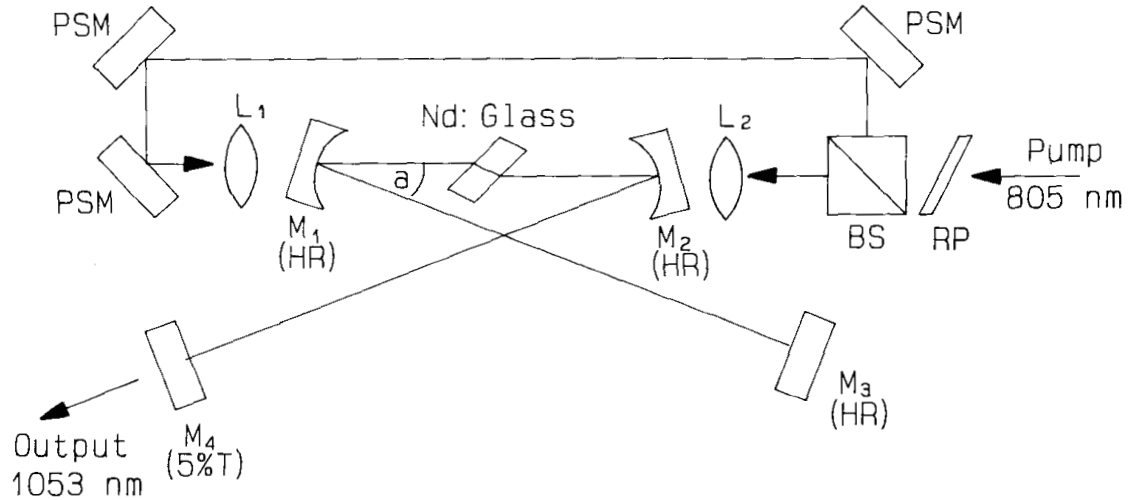

Fig. 6. Ti : sapphire pumped Nd: glass laser. PSM: Pump steering mirror. RP: Retardation plate. BS: Beam splitter. Angle (a): $16.8^{\circ}$.

size was measured to be $<24 \mu \mathrm{m}$ in both the vertical and horizontal planes. The output coupling was $5 \%$. This output coupling was not optimized for maximum output power.

The performance of this laser when pumped with four laser diodes is shown in Fig. 5. The laser threshold is 50 $\mathrm{mW}$ of absorbed pump power, and the slope efficiency $32.4 \%$. The maximum output power was $415 \mathrm{~mW}$ for an absorbed pump power of $1.4 \mathrm{~W}$. There was no decrease of laser output power with time, and the laser output was at all times $\mathrm{TEM}_{00}$. As stated previously, thermally induced birefringence can cause a decrease in slope efficiency due to depolarization of the intracavity beam and increased loss from the Brewster surfaces. We monitored the Brewster reflections as a function of pump power, and observed the expected quadratic dependence of reflectivity with pump power consistent with losses due to thermally induced birefringence [18]. However, at maximum pump power, the reflected signal amounted to no more than $4 \%$ of the laser output power, demonstrating that in this laser, thermally induced birefringence introduces negligible losses. This is further confirmed by the fact that no evidence was observed of a decrease in slope efficiency with increasing pump power. The laser output was found to be very well polarized. When passed through a polarizing beam splitter, the ratio of light transmitted to that rejected was $\sim 2000: 1$.

We have compared the laser-diode-pumped performance of the $\mathrm{Nd}$ : glass laser with that of a $\mathrm{Ti}$ : sapphire pumped system. The laser cavity arrangement for the Ti: sapphire pumped Nd:glass laser was slightly modified in order to facilitate switching from a standing wave configuration to the ring configuration required for single frequency operation (see Section IV). The standing wave cavity configuration is shown in Fig. 6.

The output of the $\mathrm{Ti}$ : sapphire laser was split into two equal intensity beams to allow symmetrical double end pumping. The beams were focused into the $\mathrm{Nd}$ : glass disk using two AR coated lenses $\left(L_{1}\right.$ and $\left.L_{2}\right)$ of focal length $7.5 \mathrm{~cm}$. The mirrors $M_{1}$ and $M_{2}$ (both $10 \mathrm{~cm} \mathrm{ROC)} \mathrm{were}$ highly reflective $(>99.9 \%)$ at the laser wavelength and highly transmissive $(>90 \%)$ at the pump wavelength. The laser resonator was completed using a highly reflective mirror $M_{3}$ and a $5 \%$ transmitting output coupler $M_{4}$.

The threshold for lasing in the Ti:sapphire pumped system was $84 \mathrm{~mW}$ of absorbed pump power $(42 \mathrm{~mW}$ in each direction). The output of the laser increased linearly with pump power, producing $480 \mathrm{~mW}$ of $\mathrm{CW}$ power for 


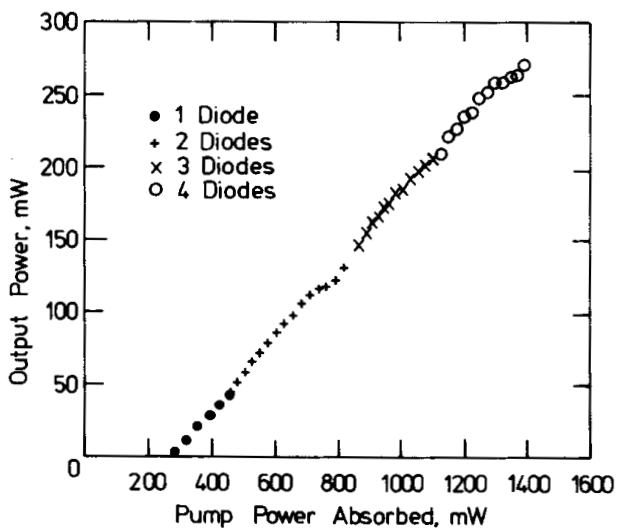

Fig. 7. Laser-diode-pumped Nd: glass laser performance with $15 \%$ output coupling.

a total incident pump power of $1.6 \mathrm{~W}$. For pump powers greater than $1.6 \mathrm{~W}$, the efficiency of the laser dropped off increasingly due to the limited power handling capability of the glass medium. Thermal damage (surface melting) was observed for powers in excess of $1 \mathrm{~W}$ incident on each surface. The optical slope efficiency of the laser was $36 \%$. The fact that this shows only a slight improvement over the laser-diode-pumped performance indicates that the non-Gaussian output from the diode lasers is not seriously affecting the $\mathrm{Nd}$ : glass laser performance.

We also investigated the performance of the diodelaser-pumped laser when the laser output coupling was increased to $15 \%$. This is of interest since as the output coupling is increased, the feedback from an external cavity will also increase, improving the chances of obtaining APM operation. The performance of the laser under these conditions is shown in Fig. 7. The laser threshold is increased to $260 \mathrm{~mW}$, and the optical slope efficiency is $23.9 \%$. The maximum output power obtained was 270 $\mathrm{mW}$. Comparing these results to the work of Krausz et al. [8], it is apparent that this system provides a viable means of obtaining subpicosecond pulses from an all solid-state laser via self-starting APM operation. Preliminary results have recently been obtained from an APM laser-diodepumped Nd:glass laser where the pulse duration was $\sim 600 \mathrm{fs}$. This is the first demonstration of this source, and full details will be presented elsewhere.

\section{Single Frequency Operation}

We have also investigated single frequency operation in the $\mathrm{Ti}$ : sapphire pumped Nd: glass laser. Several techniques have been developed to achieve single longitudinal mode operation from solid state lasers, including the use of short standing wave resonators [19] and intracavity etalons [20]. The use of ring lasers, where one of the two counter propagating traveling waves is suppressed, has also been widely reported, in particular the development of the nonplanar ring oscillator (NPRO) [21]. This suppression causes unidirectional oscillation and so overcomes spatial hole burning which would otherwise pro- mote multimode oscillation. The approach taken in our experiment involved using a traveling wave acoustooptic modulator ( $Q$ switch) to introduce a differential loss between the counterpropagating beams in a ring laser. This technique was employed recently to obtain single frequency operation in a diode-pumped Nd: YAG laser [22], and has several advantages over the other techniques. In particular, much higher power operation can be obtained than from the very short, standing wave cavities, and the technique is simpler to implement than the NPRO. Also, the acoustooptic modulator can act as a $Q$ switch if desired, and the technique can be used for those laser materials which do not have a large enough Verdet constant to achieve single frequency operation by applying a magnetic field to the laser material itself. Additionally, this method can be applied to laser materials exhibiting birefringence, such as Nd: YLF [23], since the unidirectional operation does not depend on polarization discrimination. The mechanism by which the modulator introduces differential loss between the two beams is not fully understood at present and requires further investigation.

The single frequency laser configuration is depicted in Fig. 8. This was arrived at by simply tilting the high reflector $M_{3}$ and output coupler in the standing wave configuration so as to complete the "figure-eight" ring path, and inserting an acoustooptic $Q$ switch at the waist formed between these two mirrors. The $Q$ switch was an AR coated plane surface lead molybdate device with an 80 $\mathrm{MHz}$ carrier frequency and a diffraction efficiency of about $0.3 \mathrm{~W}^{1 / 2}$

Unidirectional oscillation was achieved by tilting the modulator about $1^{\circ}$ away from the Bragg diffraction angle where maximum diffraction efficiency occurs and applying $1 \mathrm{~W}$ of drive RF power. This provided sufficient differential loss to maintain single frequency operation up to $60 \mathrm{~mW}$ of laser output power. The corresponding pump power was $800 \mathrm{~mW}$. It was noted that increasing the pump power above $800 \mathrm{~mW}$ required increasing the $Q$-switch diffraction power to prevent multimode operation. This acted to clamp the maximum power available in one beam. Furthermore, by increasing the diffraction power of the modulator, the cavity finesse was impaired, resulting in poorer gain extraction and subsequently a greater likelihood of thermal damage in the glass laser medium.

The linewidth of the laser was measured in a variety of ways. An optical spectrum analyzer $(300 \mathrm{MHz}$ free-spectral range confocal interferometer) indicated stable single frequency operation with the $Q$ switch activated, with a mechanical frequency jitter of $8 \mathrm{MHz}$ (not actively stabilized) and an instantaneous linewidth limited by the resolution of the analyzer at $2 \mathrm{MHz}$. A higher resolution measurement of the instantaneous linewidth was obtained using a homodyne scheme incorporating a $4.6 \mathrm{~km}$ fiber delay line. The measured linewidth of $60 \pm 10 \mathrm{kHz}$ yields a deconvolved linewidth of $32 \pm 5.3 \mathrm{kHz}$. This was sufficiently close to the estimated resolution of $40 \mathrm{kHz}$, determined by the length of the delay line, that it has to be considered an upper limit on the laser linewidth [24]. 


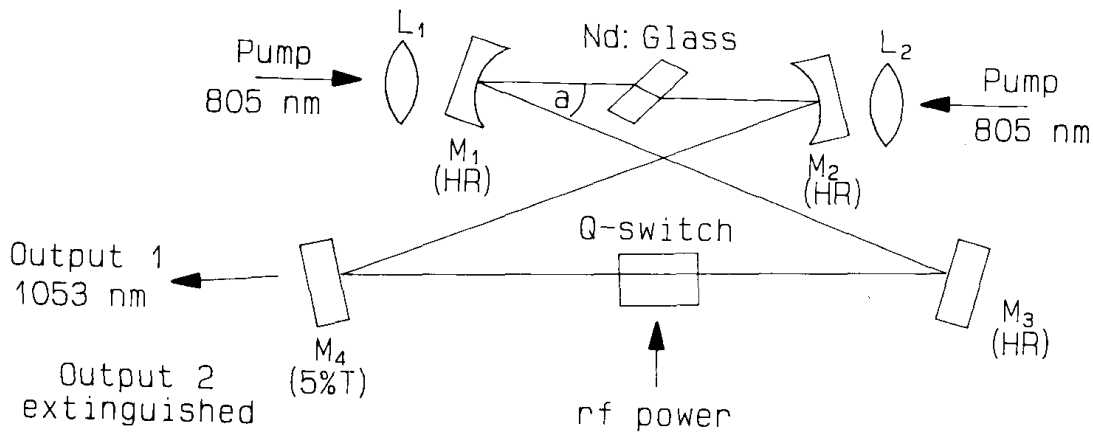

Fig. 8. Single frequency Nd: glass laser using an acoustooptic $Q$ switch to force unidirectional oscillation.

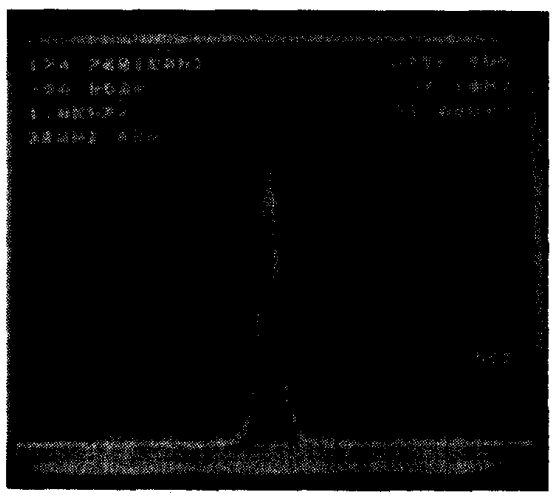

Fig. 9. Oscilloscope trace of intermode beat during dual mode operation. Deconvolution of the total signal bandwidth of $400 \mathrm{~Hz}$ indicates a single mode linewidth of around $150 \mathrm{~Hz}$ (scope resolution $=300 \mathrm{~Hz}$ ).

To further improve the resolution of this measurement we have employed a novel technique based on dual mode oscillation. Dual mode oscillation (lasing on a pair of longitudinal modes of the laser resonator) was achieved with the laser running unidirectionally by misaligning the resonator slightly and reducing the RF power to the $Q$ switch so that bidirectional (multimode) oscillation was just held off. Under these conditions, a pair of modes experiencing the same gain in the laser would oscillate until thermal drifting through the gain spectrum caused a jump to a different pair of modes. The two modes were not always adjacent in frequency, suggesting a shaping of the laser gain profile by residual etalon effects in the laser.

To measure the linewidth of an individual mode, the intermode beat was monitored with a fast photodiode and RF spectrum analyser at times when the two oscillating modes were adjacent. Deconvolving the beat signal (assuming a Gaussian frequency profile) gave a value for the longitudinal mode bandwidth and hence an upper limit on the linewidth of the laser when running single frequency (some broadening of the linewidth is expected due to the reduction in cavity finesse required to attain dual mode oscillation). Using this method, the instantaneous linewidth of the single frequency laser was found to be less than $200 \mathrm{~Hz}$, the measurement being impaired by the 300
$\mathrm{Hz}$ minimum resolution of our RF spectrum analyzer. A plot of the intermode beat is shown in Fig. 9, indicating a total convolved signal bandwidth of $400 \mathrm{~Hz}$, corresponding to an actual laser linewidth of around $150 \mathrm{~Hz}$.

\section{Summary}

We have reported the active mode locking of a laserdiode-pumped $\mathrm{Nd}$ : glass laser. Using an acoustooptic amplitude modulator, a minimum pulse duration of 9 ps was obtained. The pulses were 1.6 times transform limited. In order to reach the powers necessary to make the laserdiode-pumped $\mathrm{Nd}$ : glass laser a viable source of sub-picosecond pulses via additive pulse mode locking, we have developed a high power $(415 \mathrm{~mW})$, high optical slope efficiency $(32.4 \%)$ system. Four broad stripe laser diodes were used to pump the laser. The performance of this laser was similar to that obtained using a Ti:sapphire laser as the pump source. Using a $\mathrm{Ti}$ : sapphire laser to simulate pumping by laser diodes, we have demonstrated a single frequency Nd: glass laser. An acoustooptic modulator was used to enforce unidirectional operation of a $\mathrm{Nd}$ : glass ring laser. We have obtained up to $60 \mathrm{~mW}$ of single frequency output, and have measured the instantaneous linewidth of the laser to be less than $300 \mathrm{~Hz}$.

\section{REFERENCES}

[1] L. Yan, P.-T. Ho, C. H. Lee, and G. L. Burdge, "Generation of ultrashort pulses from a neodymium glass laser system," IEEE $J$. Quantum Electron., vol. 25, pp. 2431-2440, 1989.

[2] W. J. Kozlovsky, T. Y. Fan, and R. L. Byer, "Diode-pumped continuous-wave Nd:glass laser," Opt. Lett., vol. 11, pp. 788-790, 1986.

[3] S. Basu and R. L. Byer, "Continuous-wave mode-locked Nd: glass laser pumped by a laser diode," Opt. Lett., vol. 13, pp. 458-460, 1988 .

[4] F. Krausz, T. Brabec, E. Winner, and A. J. Schmidt, "Mode locking of a continuous wave $\mathrm{Nd}$ : glass laser pumped by a multistripe diode laser," Appl. Phys. Lett., vol. 55, pp. 2386-2388, 1989.

[5] T. E. Dimmick, "Semiconductor-laser-pumped, cw mode-locked $\mathrm{Nd}$ : phosphate glass laser oscillator and regenerative amplifier,'” Opt. Lett., vol. 15, pp. 177-179, 1990.

[6] D. W. Hughes, J. R. M. Barr, and D. C. Hanna, "Mode locking of a diode-laser-pumped $\mathrm{Nd}$ : glass laser by frequency modulation," Opt. Lett., vol. 16, pp. 147-149, 1991.

[7] J. Korn, T. H. Jeys, and T. Y. Fan, " $\mathrm{CW}$ operation of a diodepumped rotating Nd : glass disk laser," in Tech. Dig., Advanced Solid State Lasers, Hilton Head, SC, paper WA3, 1991. 
[8] F. Krausz, C. Spielmann, T. Brabec, E. Wintner, and A. J. Schmidt, "Self-starting additive-pulse mode locking of a Nd : glass laser," Opt. Lett., vol. 15, pp. 1082-1084, 1990.

[9] R. Roy, P. A. Schulz, and A. Walther, "Acousto-optic modulator as an electrically selectable unidirectional device in a ring laser," $O p t$ Lett., vol. 12, pp. 672-674, 1987.

[10] H. W. Kogelnik, E. P. Ippen, A. Dienes, and C. V. Shank, "Astigmatically compensated cavities for CW dye lasers," IEEE J. Quantum Electron., vol. QE-8, pp. 373-379, 1972.

[11] D. W. Hughes, J. R. M. Barr, and D. C. Hanna, "A high power, high efficiency, laser-diode-pumped, continuous wave miniature Nd: glass laser," Opt. Commun., vol. 84, pp. 401-408, 1991; see also — , "A high-efficiency, laser-diode-pumped continuous wave miniature Nd : glass laser," in Tech. Dig., Conf. Lasers and ElectroOpt., Baltimore, MD, paper CME6, 1991.

[12] G. T. Maker, S. J. Keen, and A. I. Ferguson, "Mode-locked and $Q$ switched operation of a diode laser pumped Nd: YAG laser operating at $1.064 \mu \mathrm{m}, "$ Appl. Phys. Lett., vol. 53, pp. 1675-1677, 1988.

[13] G. T. Maker and A. I. Ferguson, "Frequency-modulation mode locking of a diode-pumped Nd: YAG laser,', Opt. Lett., vol. 14, pp. 788$790,1989$.

[14] C. S. Adams, G. T. Maker, and A. I. Ferguson, "FM operation of Nd: YAG lasers with standing wave and ring cavity configurations," Opt. Commun., vol. 76, pp. 127-130, 1990.

[15] J. R. M. Barr and D. W. Hughes, "Coupled cavity modelocking of a Nd : YAG laser using second-harmonic generation," Appl. Phys. B vol. B49, pp. 323-325, 1989

[16] J. Goodberlet, J. Jacobson, J. G. Fujimoto, P. A. Schulz, and T. Y. Fan, "Self-starting additive-pulse mode-locked diode-pumped Nd: YAG laser,"' Opt. Lett., vol. 15, pp. 504-506, 1990.

[17] G. P. A. Malcolm, P. F. Curley, and A. I. Ferguson, "Additive pulse mode locking of a diode-pumped Nd: YLF laser," Opt. Lett., vol. 15, pp. 1303-1305, 1990

[18] W. Koechner, Solid-State Laser Engineering. Berlin: Springer-Verlag, 1988, ch. 7, pp. 361-368.

[19] J. J. Zayhowski and A. Mooradian, " Single-frequency microchip Nd lasers," Opt. Lett., vol. 14, pp. 24-26, 1989.

[20] P. Nachman, J. Munch, and R. Yee, "Diode-pumped, frequencystable, tunable, continuous-wave Nd : glass laser," IEEE J. Quantum Electron., vol. 26, pp. 317-322, 1990

[21] T. J. Kane and R. L. Byer, "Monolithic, unidirectional single-mode Nd: YAG ring laser," Opt. Lett., vol. 10, pp. 65-67, 1985.

[22] W. A. Clarkson and D. C. Hanna, "Acousto-optically induced unidirectional single mode operation of a $Q$-switched miniature Nd:YAG ring laser,"'Opt. Commun., vol. 81, pp. 375-378, 1991.

[23] W. A. Clarkson and D. C. Hanna, "Single frequency $Q$-switched operation of a diode-pumped Nd: YLF ring laser,' Opt. Commun., vol. 84 , pp. 51-54, 1991 .

[24] G. Cowle, "Laser linewidth determination by the self heterodyne technique," Telecom Australia Research Lab. Rep. 7919, 1988

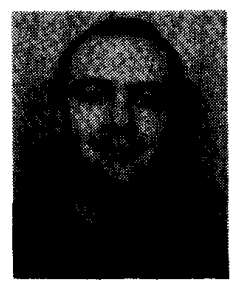

David W. Hughes was born in Bristol, U.K., in 1964. He received the B.Sc. degree in physics from the University College of Swansea in 1985

Between 1985 and 1988 he was a research physicist with British Aerospace, Sowerby Research Centre, Bristol, where his work was concerned with the development of $\mathrm{CO}_{2}$ lasers. Between 1988 and 1991 he was a $\mathrm{Ph} . \mathrm{D}$. student with the $\mathrm{De}$ partment of Physics at Southampton University His doctoral work was concerned with the devel opment of short pulse generation from Nd:YAG and Nd: glass lasers. He is currently a Research Assistant at the OptoElectronics Research Centre, Southampton University, where his interest are in diode laser pumping of, and short pulse generation from, Nd-doped lasers.

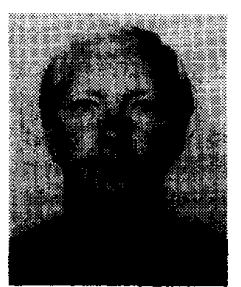

Mark W. Phillips was born in Merthyr Tydfil Wales, in 1963. He received the B.Sc. degree in physics from Southampton University in 1985 and the Ph.D. degree in 1989. His doctoral work concerned the development of rare-earth-doped optical fiber lasers.

From 1989 to 1991 he held an SERC research fellowship with the Department of Physics, Southampton University, to investigate novel techniques for generating ultrashort pulses from optical fiber lasers. He is currently a research fellow of the Opto-Electronics Research Centre, Southampton University, en gaged in the study of nonlinear optical pulse generation in miniature solidstate lasers.

Dr. Phillips is a member of the Institute of Physics.

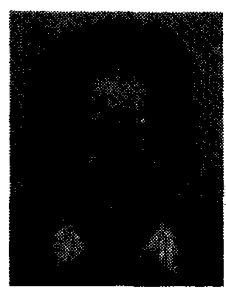

John R. M. Barr received the B. Sc degree in physics (first class) from St. Andrews University, Scotland, in 1982, and the Ph.D. degree from the University of Southampton, U.K., in 1986.

Between 1985 and 1988 he was with the Central Laser Facility, Rutherford Appleton Laboratory, U.K, as part of the Laser Development group. Since 1988 he has held a lectureship with the Department of Physics, Southampton University. He is also a research lecturer within the Opto-Electronics Research Centre, Southampton University. His research interests include laser physics, particularly techniques for generation of short pulses in laser diode pumped solid-state lasers, and the laser spectroscopy of fundamental systems including hydrogen and muonium.

Dr. Barr is the secretary to the Quantum Electronics group of the Institute of Physics and is a member of the Institute of Physics and the Optical Society of America.

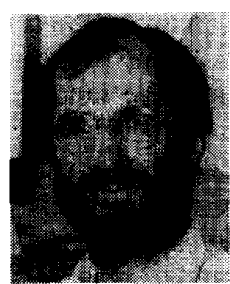

David C. Hanna was born in Nottingham, England, in 1941. He received the B.A. degree from Cambridge University in 1962 and the Ph.D. degree from Southampton University in 1967.

Currently, he is a Professor with the Department of Physics and a Deputy Director of the Optoelectronics Research Centre, Southampton University. He has spent periods of leave at the Politechnico di Milano, Italy, and, as an Alex ander von Humboldt Fellow, at the University of Munich, Munich, Germany. He is a past chairma of the Quantum Electronics Group of the Institute of Physics. His present interests include optical fiber lasers, diode-pumped solid-state lasers, crystal waveguide lasers, and optical parametric oscillators. 